\title{
Efficient Two-Step Synthesis of 9-Aryl-6-hydroxy-3H-xanthen-3-one Fluorophores
}

\author{
James P. Bacci, Aaron M. Kearney and David L. Van Vranken* \\ Department of Chemistry, University of California, Irvine, CA 92697-2025
}

\section{SUPPORTING INFORMATION}

\section{Experimental Procedures}

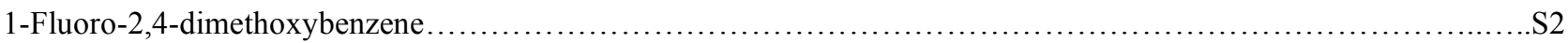

[(4-Formyl-2-methoxy-phenyl)-methoxycarbonylmethyl-amino]-acetic acid methyl ester (1c) ....................S2

(\{4-[Bis-(5-fluoro-2,4-dihydroxy-phenyl)-methyl]-2-methoxy-phenyl $\}$-methoxycarbonylmethyl-amino)-acetic acid

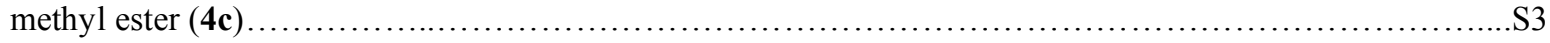

4-Fluoro-6-((5-fluoro-2,4-dihydroxyphenyl)(phenyl)methyl)benzene-1,3-diol (4d) .............................S4

4-((4-Dimethylamino)phenyl)(5-fluoro-2,4-dihydroxyphenyl)methyl)-6-flourobenzene-1,3-diol (4e).................S4

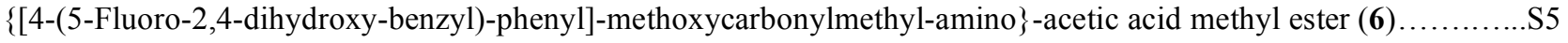

$\{[4-(2,7-D i f l u o r o-6-h y d r o x y-3-o x o-3 H$-xanthen-9-yl)-2-methoxy-phenyl]-methoxycarbonylmethyl-amino $\}$ -

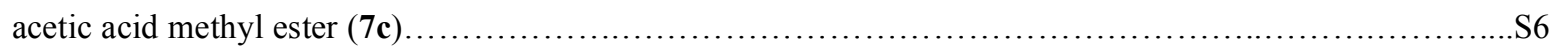

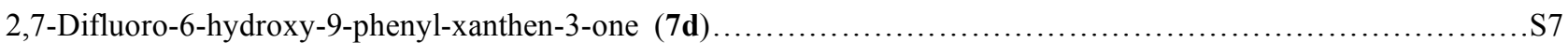

\section{${ }^{1} \mathrm{H}-,{ }^{13} \mathrm{C}$ - and ${ }^{19}$ F-NMR Spectra}

[(4-Formyl-2-methoxy-phenyl)-methoxycarbonylmethyl-amino]-acetic acid methyl ester (1c)

S8-S9

(\{4-[Bis-(5-fluoro-2,4-dihydroxy-phenyl)-methyl]-phenyl $\}$-methoxycarbonylmethyl-amino)-

acetic acid methyl ester $(\mathbf{4 b})$.

(\{4-[Bis-(5-fluoro-2,4-dihydroxy-phenyl)-methyl]-2-methoxy-phenyl\}-methoxycarbonylmethyl-amino)-acetic acid methyl ester (4c).

4-Fluoro-6-((5-fluoro-2,4-dihydroxyphenyl)(phenyl)methyl)benzene-1,3-diol (4d).

4-((4-Dimethylamino)phenyl)(5-fluoro-2,4-dihydroxyphenyl)methyl)-6-flourobenzene-1,3-diol (4e)

(\{4-[[4-Bis-methoxycarbonylmethyl-amino)-phenyl]-(5-fluoro-2,4-dihydroxy-phenyl)-methyl]-phenyl\}methoxycarbonylmethyl-amino)-acetic acid (5).

\{[4-(5-Fluoro-2,4-dihydroxy-benzyl)-phenyl]-methoxycarbonylmethyl-amino $\}$-acetic acid methyl ester (6).

$\{[4-(2,7-D i f l u o r o-6-h y d r o x y-3-o x o-3 H$-xanthen-9-yl)-phenyl]-methoxycarbonylmethyl-amino $\}$ acetic acid methyl ester (7b)

\{[4-(2,7-Difluoro-6-hydroxy-3-oxo-3H-xanthen-9-yl)-2-methoxy-phenyl]-methoxycarbonylmethyl-amino\}acetic acid methyl ester $(\mathbf{7 c})$ 


\section{Experimental Procedures}

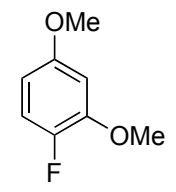

1-Fluoro-2,4-dimethoxybenzene. SelectFluor ${ }^{\circledR}(4.35 \mathrm{~g}, 12.28 \mathrm{mmol})$ was dissolved in $20 \mathrm{~mL} \mathrm{MeCN}$ containing $2 \mathrm{~mL}$ of $\mathrm{H}_{2} \mathrm{O}$. To this cloudy solution was added 1,3-dimethoxybenzene (3.22 mL, 24.56 mmol) and the reaction mixture was heated to $50{ }^{\circ} \mathrm{C}$ and stirred for $30 \mathrm{~min}$. After cooling to room temperature, the reaction was diluted with $\mathrm{H}_{2} \mathrm{O}(20 \mathrm{~mL})$ and organics extracted with $\mathrm{Et}_{2} \mathrm{O}(2 \times 75 \mathrm{~mL})$. The $\mathrm{Et}_{2} \mathrm{O}$ layer was dried with $\mathrm{MgSO}_{4}$, filtered and concentrated in vacuo. The crude light brown oil was purified by silica gel flash chromatography with $2.5 \% \mathrm{Et}_{2} \mathrm{O} /$ hexanes as eluant to give 1-fluoro-2,4dimethoxybenzene as a clear oil (1.31 g, 68\%).

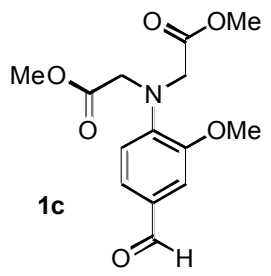

[(4-Formyl-2-methoxy-phenyl)-methoxycarbonylmethyl-amino]-acetic acid methyl ester (1c). To a flame-dried flask equipped with a magnetic stir bar was added $10 \mathrm{~mL}$ anhydrous DMF. The flask was flushed with argon and cooled in an ice-water bath. To the stirring DMF at $0{ }^{\circ} \mathrm{C}$ was added dropwise $\mathrm{POCl}_{3}(5.0 \mathrm{~mL}, 53.50 \mathrm{mmol})$. After stirring for $10 \mathrm{~min}$, [methoxycarbonylmethyl-(2-methoxy-phenyl)amino]-acetic acid methyl ester $(1.43 \mathrm{~g}, 5.35 \mathrm{mmol})$ was added dropwise as a solution in $10 \mathrm{~mL}$ anhydrous DMF. The resulting yellow solution was allowed to warm to room temperature and then heated to $100{ }^{\circ} \mathrm{C}$ for $30 \mathrm{~min}$. After cooling to room temperature, the reaction mixture was poured into $200 \mathrm{~mL}$ of ice water. The $\mathrm{pH}$ was adjusted to $\sim 7$ by addition of solid $\mathrm{NaHCO}_{3}$ and organics extracted with EtOAc $(3 \times 200 \mathrm{~mL})$. The combined organic extracts were dried with $\mathrm{MgSO}_{4}$, filtered and concentrated in vacuo. The crude 
brown oil was purified by silica gel flash chromatography (50\% EtOAc/hexanes) to give 1c as a viscous light yellow oil (1.42 g, 90\%): ${ }^{1} \mathrm{H}$ NMR (400 MHz, $d_{6}$-DMSO, $\left.298 \mathrm{~K}\right) \delta 9.75(\mathrm{~s}, 1 \mathrm{H}), 7.40(\mathrm{~d}, J=8.5 \mathrm{~Hz}$, $1 \mathrm{H}), 7.34(\mathrm{~s}, 1 \mathrm{H}), 6.72(\mathrm{~d}, J=8.3 \mathrm{~Hz}, 1 \mathrm{H}), 4.21(\mathrm{~s}, 4 \mathrm{H}), 3.74(\mathrm{~s}, 3 \mathrm{H}), 3.67(\mathrm{~s}, 6 \mathrm{H}) ;{ }^{13} \mathrm{C}$ NMR $(125$ MHz, $d_{6}$-DMSO, $\left.298 \mathrm{~K}\right) \delta 190.6,170.8,149.4,144.4,128.8,125.6,115.7,111.1,55.8,54.0,51.6 ; \quad$ IR (thin film) 2956, 2835, 2735, 1749, 1682, 1597, 1398, 1263, 1204, 1165, $1135 \mathrm{~cm}^{-1}$; TLC R $\mathrm{R}_{f}=0.50(60 \%$ EtOAc/hexanes); LRMS (ESI) $\mathrm{m} / \mathrm{z}$ (relative intensity): 236(5), 318(100); HRMS (CI) $\mathrm{m} / \mathrm{z}$ calcd for $\mathrm{C}_{14} \mathrm{H}_{17} \mathrm{NO}_{6} \mathrm{Na}[\mathrm{M}+\mathrm{Na}]^{+}, 318.0954$ found 318.0949.

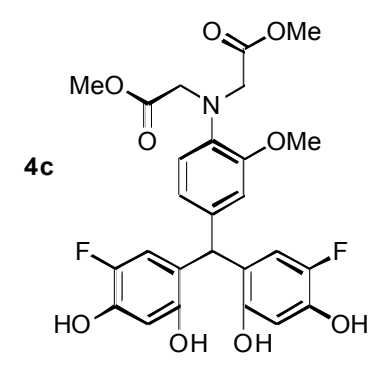

(\{4-[Bis-(5-fluoro-2,4-dihydroxy-phenyl)-methyl]-2-methoxy-phenyl\}methoxycarbonylmethyl-amino)-acetic acid methyl ester (4c). As described in the general procedure, 4-fluororesorcinol (50 mg, $0.390 \mathrm{mmol})$ and aldehyde $1 \mathrm{c}(58 \mathrm{mg}, 0.195 \mathrm{mmol})$ were stirred for $2 \mathrm{~h}$ and subjected to the work-up procedure. Purification by silica gel flash chromatography (15-35\% acetone/toluene $+1 \% \mathrm{AcOH})$ gave $4 \mathbf{c}$ as an orange/brown powder $(85 \mathrm{mg}, 81 \%)$ : $\mathrm{mp} 180-182{ }^{\circ} \mathrm{C}$ (toluene); ${ }^{1} \mathrm{H}$ NMR (400 MHz, $d_{6}$-DMSO, $\left.298 \mathrm{~K}\right) \delta 9.43(\mathrm{~s}, 2 \mathrm{H}), 9.01(\mathrm{~s}, 2 \mathrm{H}), 6.56(\mathrm{~d}, J=8.2 \mathrm{~Hz}, 1 \mathrm{H})$, $6.51(\mathrm{~d}, J=1.7 \mathrm{~Hz}, 1 \mathrm{H}), 6.41(\mathrm{~d}, J=7.9 \mathrm{~Hz}, 2 \mathrm{H}), 6.35(\mathrm{dd}, J=1.7,8.2 \mathrm{~Hz}, 1 \mathrm{H}), 6.26(\mathrm{~d}, J=12.4 \mathrm{~Hz}, 2$ H), $5.63(\mathrm{~s}, 1 \mathrm{H}), 4.04(\mathrm{~s}, 4 \mathrm{H}), 3.62(\mathrm{~s}, 6 \mathrm{H}), 3.56(\mathrm{~s}, 3 \mathrm{H}) ;{ }^{13} \mathrm{C}$ NMR $\left(125 \mathrm{MHz}, d_{6}\right.$-DMSO, $\left.298 \mathrm{~K}\right) \delta$ 171.4, 150.6, 150.0, 143.7 (d, $J=225 \mathrm{~Hz}), 142.7(\mathrm{~d}, J=13 \mathrm{~Hz}), 136.6(\mathrm{~d}, J=63 \mathrm{~Hz}), 120.9(\mathrm{~d}, J=25$ $\mathrm{Hz}$ ), 117.2, 115.8, 113.3, 104.4, 55.6, 53.4, 51.3, 43.1; IR (thin film) 3315.7, 3164.4, 2355.8, 2334.2, 1752.7, 1626.3, 1508.1, $1438.2 \mathrm{~cm}^{-1} ;$ TLC R $\mathrm{R}_{f}=0.25$ (50\% acetone/toluene $+1 \%$ AcOH); LRMS (ESI) $\mathrm{m} / \mathrm{z}$ (relative intensity): 534(8), 556(100); HRMS (CI) $m / z$ calcd for $\mathrm{C}_{26} \mathrm{H}_{25} \mathrm{~F}_{2} \mathrm{NO}_{9} \mathrm{Na}[\mathrm{M}+\mathrm{Na}]^{+}, 556.1395$, found 556.1406 . 


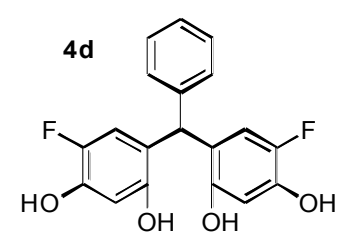

4-Fluoro-6-((5-fluoro-2,4-dihydroxyphenyl)(phenyl)methyl)benzene-1,3-diol (4d). As described in the general procedure, 4-fluororesorcinol (50 mg, $0.390 \mathrm{mmol})$ and benzaldehyde $(19.8 \mu \mathrm{L}$, $0.195 \mathrm{mmol})$ were stirred for $2 \mathrm{~h}$. Purification by silica gel flash chromatography (10-30\% acetone/toluene $+1 \% \mathrm{AcOH})$ gave $4 \mathbf{e}$ as a yellow powder $(57 \mathrm{mg}, 85 \%): \mathrm{mp} \mathrm{202-205}{ }^{\circ} \mathrm{C}$ (toluene); ${ }^{1} \mathrm{H}$ NMR (500 MHz, $d_{6}$-DMSO, $\left.298 \mathrm{~K}\right) \delta 9.47$ (s, $\left.2 \mathrm{H}\right), 9.06$ (s, $\left.2 \mathrm{H}\right), 7.27-7.22(\mathrm{~m}, 2 \mathrm{H}), 7.18-7.14(\mathrm{~m}, 1 \mathrm{H})$, 6.97-6.94 (m, $2 \mathrm{H}), 6.43(\mathrm{~d}, J=7.9 \mathrm{~Hz}, 2 \mathrm{H}), 6.25(\mathrm{~d}, J=12.5 \mathrm{~Hz}, 2 \mathrm{H}), 5.73(\mathrm{~s}, 1 \mathrm{H}) ;{ }^{13} \mathrm{C}$ NMR $(125$ MHz, $d_{6}$-DMSO, $\left.298 \mathrm{~K}\right) \delta 151.4,144.7(\mathrm{~d}, J=228 \mathrm{~Hz}), 144.7,143.6$ (d, $\left.J=13 \mathrm{~Hz}\right), 129.4,128.7,126.4$, 121.4, 116.7 (d, $J=25 \mathrm{~Hz}), 105.1,42.4$; IR (thin film) 3130.2 (v. br.), 2257.4, 1633.6, 1537.9, 1454.8, $1192.7 \mathrm{~cm}^{-1} ;$ TLC $\mathrm{R}_{f}=0.20$ (20\% acetone/toluene $\left.+1 \% \mathrm{AcOH}\right) ; \mathrm{LRMS}(\mathrm{ESI}) \mathrm{m} / z$ (relative intensity): 217(100) (fragmentation from $\mathrm{MeSO}_{3} \mathrm{H}$ ); HRMS (CI) $\mathrm{m} / z$ calcd for $\mathrm{C}_{13} \mathrm{H}_{10} \mathrm{FO}_{2}[\mathrm{M}]^{+}, 217.0665$ found 217.0673 .

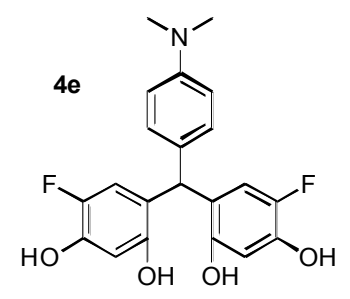

4-((4-Dimethylamino)phenyl)(5-fluoro-2,4-dihydroxyphenyl)methyl)-6-flourobenzene-1,3-diol (4e). As described in the general procedure, 4-fluororesorcinol (50 mg, $0.390 \mathrm{mmol})$ and 4dimethylaminobenzaldeyhde $(29 \mathrm{mg}, 0.195 \mathrm{mmol})$ were stirred for $2 \mathrm{~h}$. The initial reaction mixture appeared to be biphasic, but became homogeneous as a dark brown precipitate formed over the $2 \mathrm{~h}$ reaction time. Upon addition of $\mathrm{NaHCO}_{3}$ in the work-up, this precipitate became soluble and the work-up was continued as usual. Purification of the crude product by silica gel flash chromatography $(20-30 \%$ acetone/toluene $+1 \% \mathrm{AcOH})$ gave $4 \mathbf{d}$ as a rose-colored powder $(53 \mathrm{mg}, 71 \%): \mathrm{mp} 183-185{ }^{\circ} \mathrm{C}$ (toluene); 
${ }^{1} \mathrm{H}$ NMR $\left(500 \mathrm{MHz}, d_{6}\right.$-DMSO, $\left.298 \mathrm{~K}\right) \delta 9.40(\mathrm{~s}, 2 \mathrm{H}), 8.96(\mathrm{~s}, 2 \mathrm{H}), 6.76(\mathrm{~d}, J=8.6 \mathrm{~Hz}, 2 \mathrm{H}), 6.62(\mathrm{~d}, J=$ $8.8 \mathrm{~Hz}, 2 \mathrm{H}), 6.40(\mathrm{~d}, J=8.0 \mathrm{~Hz}, 2 \mathrm{H}), 6.25(\mathrm{~d}, J=12.5 \mathrm{~Hz}, 2 \mathrm{H}), 5.61(\mathrm{~s}, 1 \mathrm{H}), 2.83(\mathrm{~s}, 6 \mathrm{H}) ;{ }^{13} \mathrm{C} \mathrm{NMR}$ $\left(125 \mathrm{MHz}, d_{6}\right.$-DMSO, $\left.298 \mathrm{~K}\right) \delta$ 150.6, 148.6, $144.0(\mathrm{~d}, J=225 \mathrm{~Hz}) ; 142.6(\mathrm{~d}, J=13 \mathrm{~Hz}), 131.5,129.2$, 121.6, 116.0 (d, $J=25 \mathrm{~Hz}), 112.3,104.4,40.7,30.4$; IR (thin film) 3418.2 (v. br.), 1636.1, 1518.4, 1443.6, $1176.2 \mathrm{~cm}^{-1} ;$ TLC $\mathrm{R}_{f}=0.30(40 \%$ acetone/toluene $+1 \%$ AcOH); LRMS (ESI) $\mathrm{m} / z$ (relative intensity): 388(100), 410(15); HRMS (CI) $m / z$ calcd for $\mathrm{C}_{21} \mathrm{H}_{20} \mathrm{~F}_{2} \mathrm{NO}_{4}[\mathrm{M}+\mathrm{H}]^{+}, 388.1360$, found 388.1361.

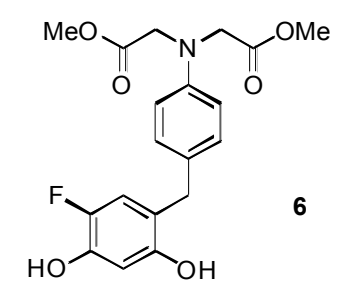

$\{[4-(5-F l u o r o-2,4-d i h y d r o x y-b e n z y l)-p h e n y l]-m e t h o x y c a r b o n y l m e t h y l-a m i n o\}-a c e t i c \quad$ acid methyl ester (6). To a flame-dried flask equipped with a magnetic stir bar was added $\mathbf{4 b}$ (50 $\mathrm{mg}, 0.100 \mathrm{mmol}$ ), $\mathrm{MeSO}_{3} \mathrm{H}(0.5 \mathrm{~mL})$ and $\mathrm{Et}_{3} \mathrm{SiH}(48 \mu \mathrm{L}, 0.300 \mathrm{mmol})$. The resulting solution was stirred for $45 \mathrm{~min}$ at room temperature, then poured into $20 \mathrm{~mL}$ of $\mathrm{H}_{2} \mathrm{O}$ containing $1 \mathrm{~g}$ of $\mathrm{NaHCO}_{3}$. The $\mathrm{pH}$ was adjusted to $\sim 5$ by addition of conc. $\mathrm{HCl}$. Organics were extracted with EtOAc $(3 \times 20 \mathrm{~mL})$, dried with $\mathrm{MgSO}_{4}$, filtered and concentrated in vacuo. The resultant yellow oil was purified by silica gel flash chromatography $(10-40 \%$ acetone/toluene $+1 \% \mathrm{AcOH})$ to give 6 as a pale yellow oil $(30.5 \mathrm{mg}, 81 \%):{ }^{1} \mathrm{H} \mathrm{NMR}\left(500 \mathrm{MHz}, d_{6^{-}}\right.$ DMSO, 298 K) $\delta 9.39$ (s, 1 H), $9.09(\mathrm{~s}, 1 \mathrm{H}), 6.97$ (d, J = 8.9 Hz, 2 H), $6.66(\mathrm{~d}, J=12.1 \mathrm{~Hz}, 1 \mathrm{H}), 6.43$ (d, $J=8.9 \mathrm{~Hz}, 2 \mathrm{H}), 6.42(\mathrm{~d}, J=7.6 \mathrm{~Hz}, 1 \mathrm{H}), 4.16(\mathrm{~s}, 4 \mathrm{H}), 3.63(\mathrm{~s}, 6 \mathrm{H}), 3.59(\mathrm{~s}, 2 \mathrm{H}) ;{ }^{13} \mathrm{C} \mathrm{NMR}(125 \mathrm{MHz}$, $d_{6}$-DMSO, $\left.298 \mathrm{~K}\right) \delta 171.2,150.7,145.7,144.4(\mathrm{~d}, J=225 \mathrm{~Hz}), 142.7(\mathrm{~d}, J=13 \mathrm{~Hz}), 129.9,129.2,118.5$, $116.4(\mathrm{~d}, J=19 \mathrm{~Hz}), 111.7,104.4,52.6,51.6,33.3$; IR (thin film) $3401.6,2987.5,1718.1,1508.2,1169.4$ $\mathrm{cm}^{-1}$; TLC $\mathrm{R}_{f}=0.58$ (30\% acetone/toluene $\left.+1 \% \mathrm{AcOH}\right) ;$ LRMS (ESI) $\mathrm{m} / z$ (relative intensity): 400(100), 777(12); HRMS (CI) $m / z$ calcd for $\mathrm{C}_{19} \mathrm{H}_{20} \mathrm{FNO}_{6} \mathrm{Na}[\mathrm{M}+\mathrm{Na}]^{+}, 400.1172$, found 400.1174 . 


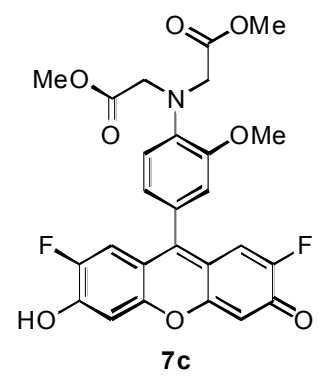

\section{$\{[4-(2,7-D i f l u o r o-6-h y d r o x y-3-0 x o-3 H$-xanthen-9-yl)-2-methoxy-phenyl]-}

methoxycarbonylmethyl-amino\}-acetic acid methyl ester (7c). As described in the general procedure,

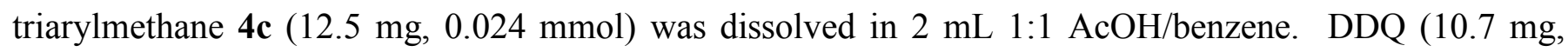
$0.048 \mathrm{mmol}$ ) was added dropwise as a solution in $1 \mathrm{~mL}$ of $1: 1 \mathrm{AcOH} /$ benzene. After stirring for $15 \mathrm{~min}$ at room temperature, the reaction mixture was concentrated in vacuo and partially purified by silica gel flash chromatography as described in the general procedure. The crude product was further purified by preparative RP-HPLC (25-60\% MeCN/0.1\% $\left.\mathrm{TFA}-\mathrm{H}_{2} \mathrm{O}\right)$ to give $7 \mathbf{c}$ as a dark brown powder $(8.4 \mathrm{mg}, 70 \%)$ : mp 282-284 ${ }^{\circ} \mathrm{C}\left(\mathrm{H}_{2} \mathrm{O}\right) ;{ }^{1} \mathrm{H}$ NMR $\left(500 \mathrm{MHz}, d_{6}\right.$-DMSO, $\left.323 \mathrm{~K}\right) \delta 7.03(\mathrm{~d}, J=1.8 \mathrm{~Hz}, 1 \mathrm{H}), 6.99(\mathrm{~d}, J=$ $11.7 \mathrm{~Hz}, 2 \mathrm{H}), 6.94(\mathrm{dd}, J=1.8,8.2 \mathrm{~Hz}, 1 \mathrm{H}), 6.88(\mathrm{~d}, J=8.2 \mathrm{~Hz}, 1 \mathrm{H}), 6.80(\mathrm{~d}, J=7.0 \mathrm{~Hz}, 2 \mathrm{H}), 4.19(\mathrm{~s}, 4$ H), $3.72(\mathrm{~s}, 3 \mathrm{H}), 3.67(\mathrm{~s}, 6 \mathrm{H}) ;{ }^{13} \mathrm{C}$ NMR $\left(125 \mathrm{MHz}, d_{6}\right.$-DMSO, $\left.323 \mathrm{~K}\right) \delta 171.7,158.8(\mathrm{~d}, J=36 \mathrm{~Hz})$, 154.1, 153.6, 151.6, 151.0, 150.5, 140.7, 124.3, 123.0, 117.7, 114.6, 112.7(d, $J=21 \mathrm{~Hz}), 105.4,56.6,54.2$, 52.0; ${ }^{19} \mathrm{~F}$ NMR (377 MHz, $d_{6}$-DMSO, $\left.298 \mathrm{~K}\right) \delta$-75.13; IR (KBr) 3396.2, 2948.8, 1688.2, 1516.1, 1301.1, $1193.5 \mathrm{~cm}^{-1}$; TLC $\mathrm{R}_{f}=0.23\left(10 \% \mathrm{MeOH} / \mathrm{CHCl}_{3}\right.$ ); LRMS (ESI) $\mathrm{m} / z$ (relative intensity): 514(100); HRMS (CI) $m / z$ calcd for $\mathrm{C}_{26} \mathrm{H}_{21} \mathrm{~F}_{2} \mathrm{NO}_{8} \mathrm{Na}[\mathrm{M}+\mathrm{Na}]^{+} 536.1133$, found 536.1121 . 


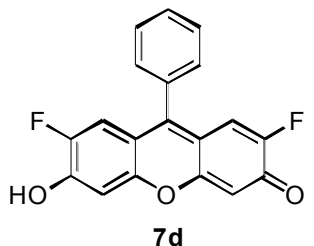

2,7-Difluoro-6-hydroxy-9-phenyl-xanthen-3-one (7d). As described in the general procedure, triarylmethane $4 \mathbf{d}(14.0 \mathrm{mg}, 0.04 \mathrm{mmol})$ was dissolved in $2.5 \mathrm{~mL} \mathrm{1:1} \mathrm{AcOH} /$ benzene. DDQ (18.0 mg, $0.08 \mathrm{mmol}$ ) was added dropwise as a solution in $2.5 \mathrm{~mL}$ of $1: 1 \mathrm{AcOH} / \mathrm{benzene}$. After stirring for $15 \mathrm{~min}$ at room temperature, the reaction mixture was concentrated in vacuo and partially purified by silica gel flash chromatography as described in the general procedure. The crude product was further purified by preparative RP-HPLC as described in the general procedure to give 7e as a dark brown powder $(5.5 \mathrm{mg}$, 39\%): $\mathrm{mp}$ dec. $300{ }^{\circ} \mathrm{C}\left(\mathrm{H}_{2} \mathrm{O}\right) ;{ }^{1} \mathrm{H}$ NMR $\left(500 \mathrm{MHz}, d_{6}\right.$-DMSO, $\left.373 \mathrm{~K}\right) \delta 7.04(\mathrm{~d}, J=9.5 \mathrm{~Hz}, 1 \mathrm{H}), 7.02(\mathrm{~d}$, $J=11.7 \mathrm{~Hz}, 2 \mathrm{H}), 6.94(\mathrm{~d}, J=8.2 \mathrm{~Hz}, 1 \mathrm{H}), 6.84(\mathrm{~d}, J=8.2 \mathrm{~Hz}, 1 \mathrm{H}), 6.81(\mathrm{~d}, J=6.3 \mathrm{~Hz}, 2 \mathrm{H}), 4.21(\mathrm{~s}, 4$ H), $3.72(\mathrm{~s}, 3 \mathrm{H}), 3.69(\mathrm{~s}, 6 \mathrm{H}) ;{ }^{13} \mathrm{C}$ NMR (125 MHz, $d_{6}$-DMSO, $\left.298 \mathrm{~K}\right) \delta$ 175.9, 175.2, 165.9, 152.0, $150.1,143.3,140.1,139.7,135.5,131.6,130.0,129.2(\mathrm{~d}, J=23 \mathrm{~Hz}), 129.0,109.4,105.2 ;{ }^{19} \mathrm{~F}$ NMR $(377$ MHz, $d_{6}$-DMSO, $\left.298 \mathrm{~K}\right) \delta$-74.48; IR (KBr) 3423.2, 1680.1, 1502.7, 1301.1, 1206.9, 1134.4, $1002.7 \mathrm{~cm}^{-1}$; TLC $\mathrm{R}_{f}=0.41\left(10 \% \mathrm{MeOH} / \mathrm{CHCl}_{3}\right)$. 


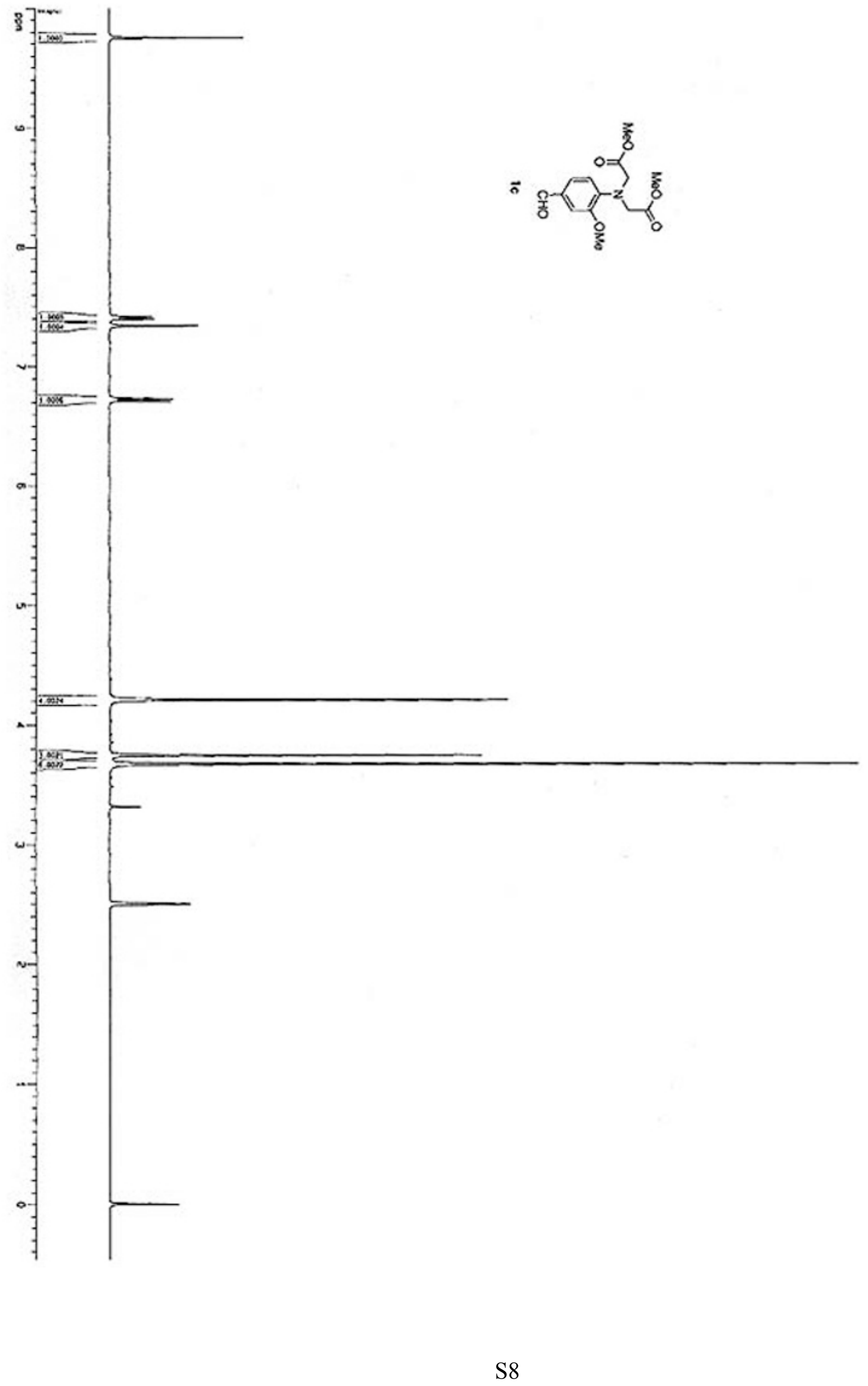




$$
L
$$




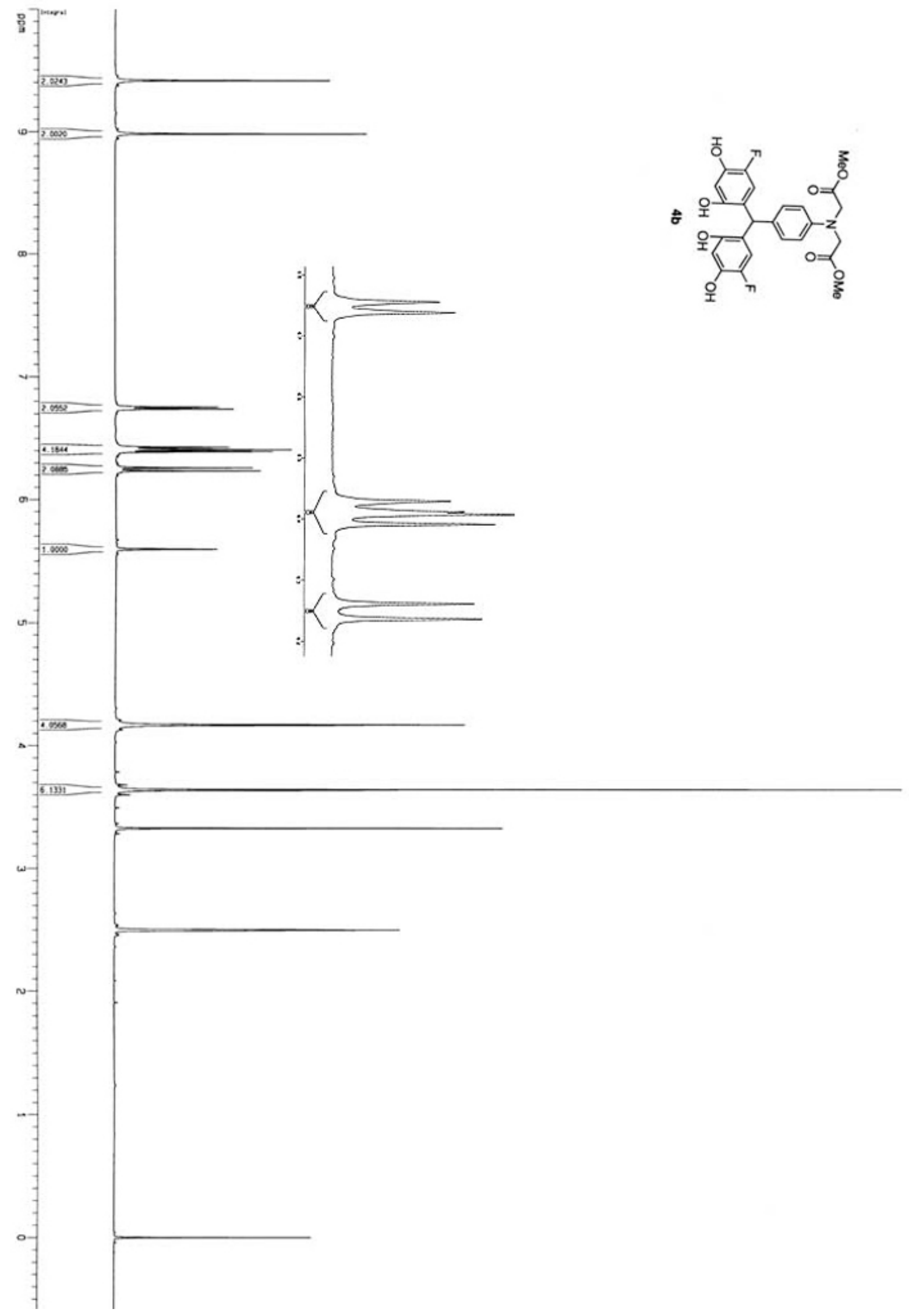




$$
\mid
$$




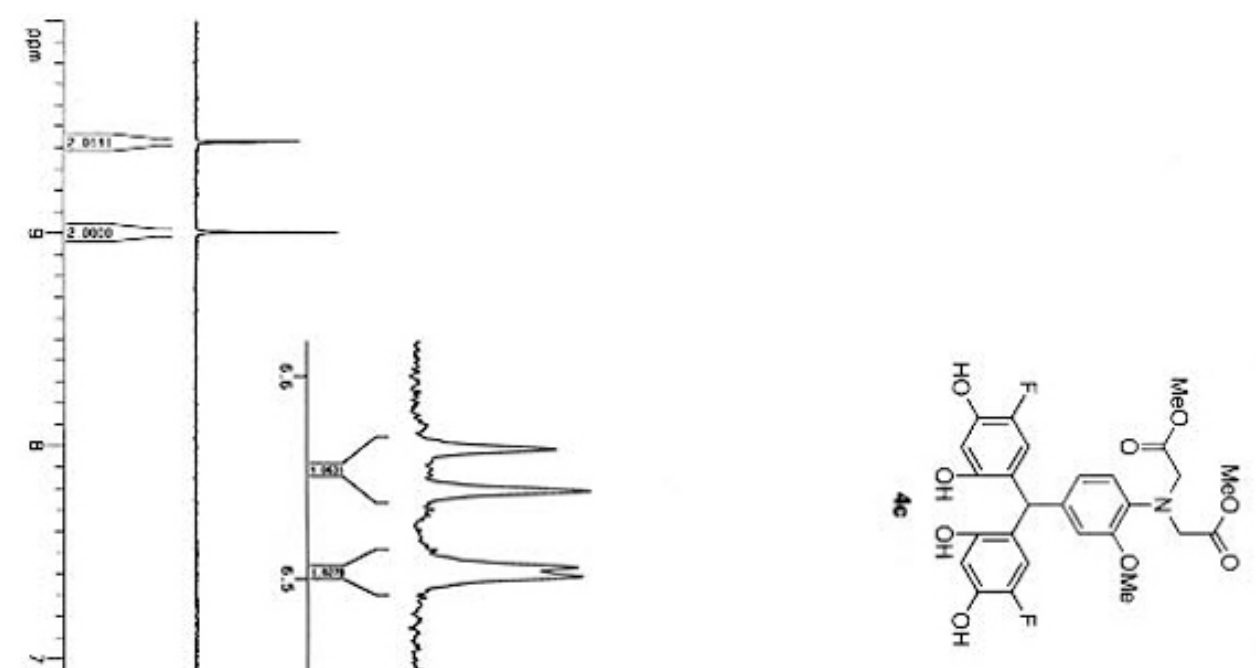

S12 


$$
\mid
$$




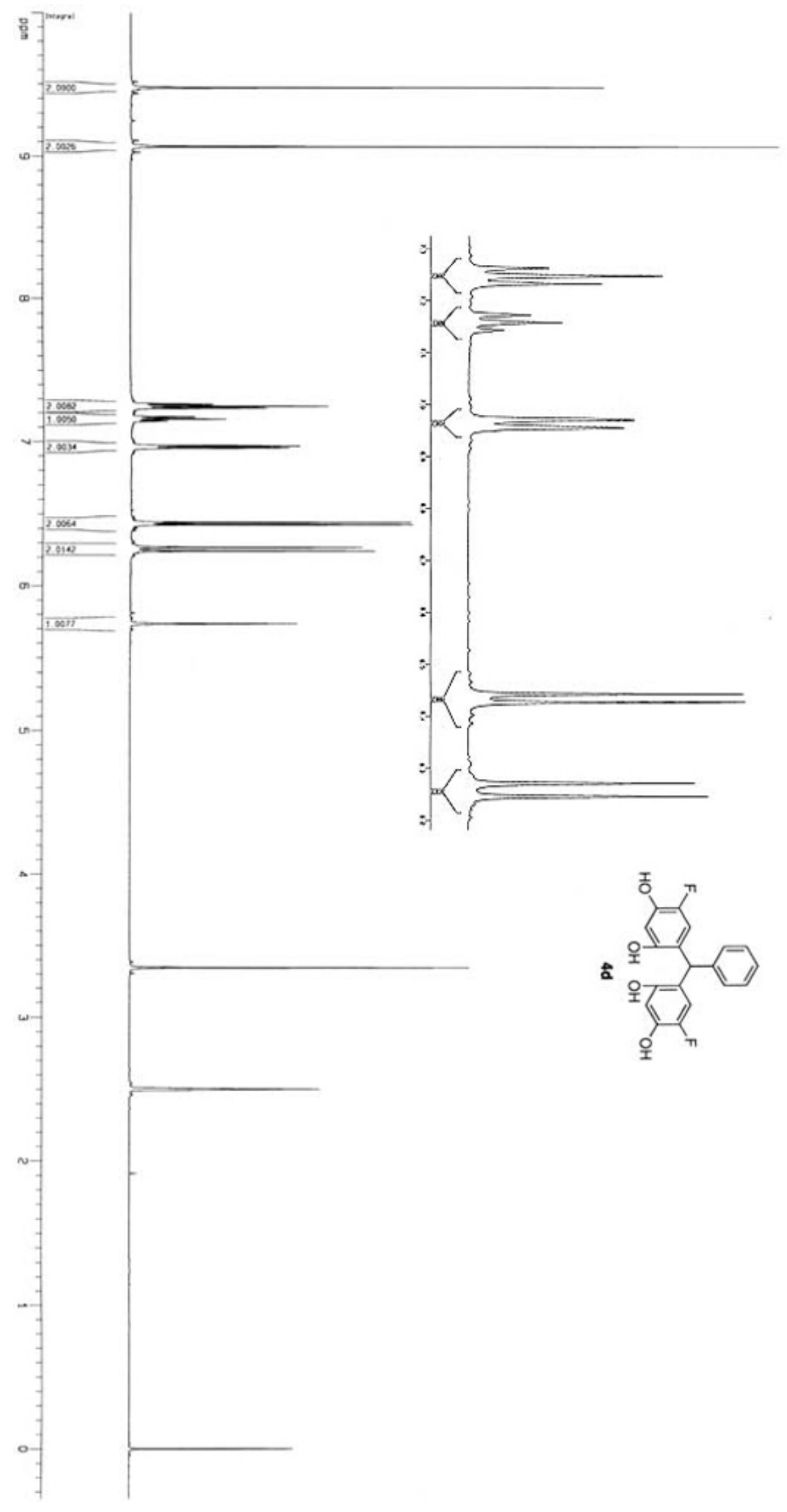




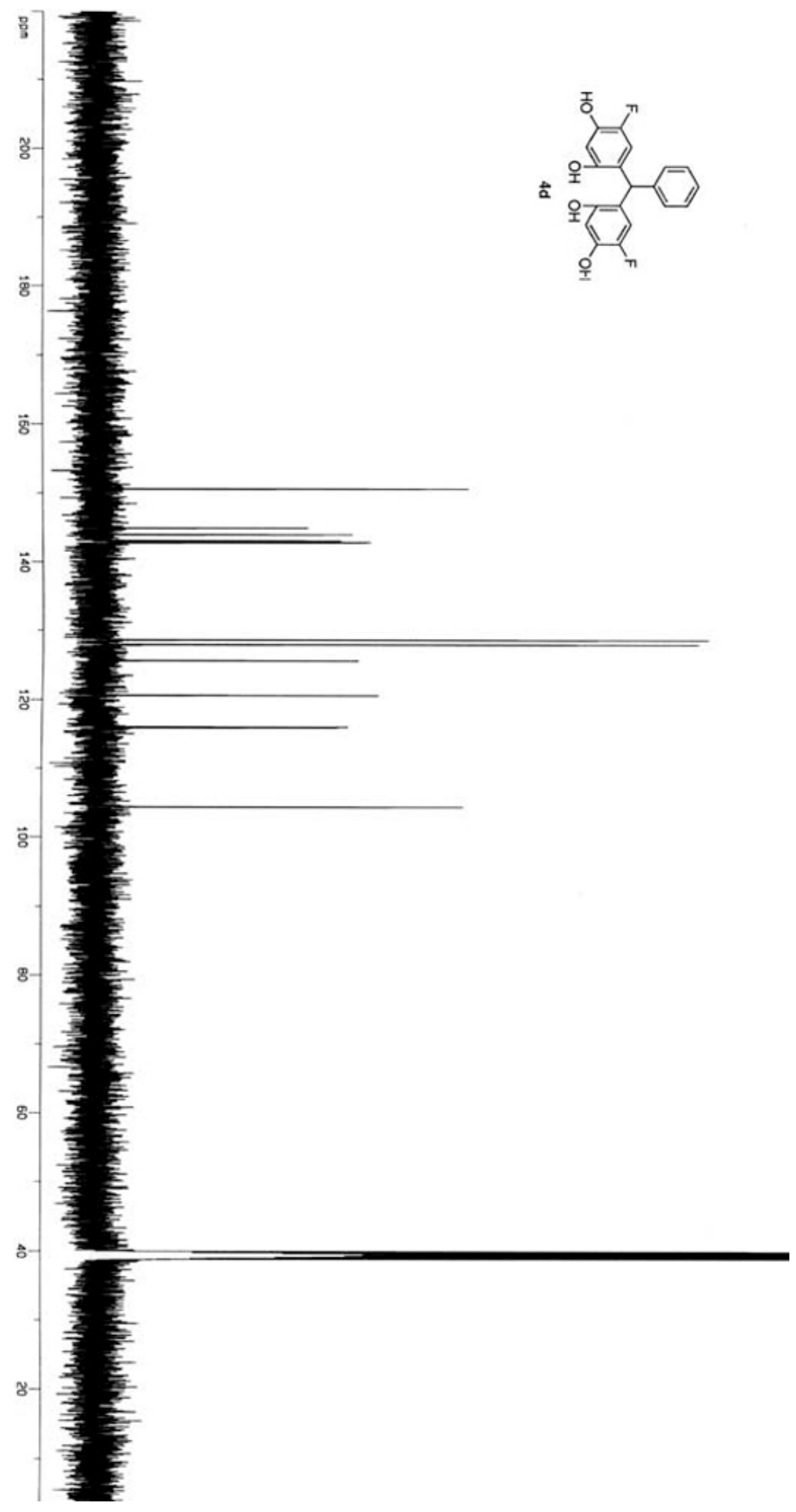




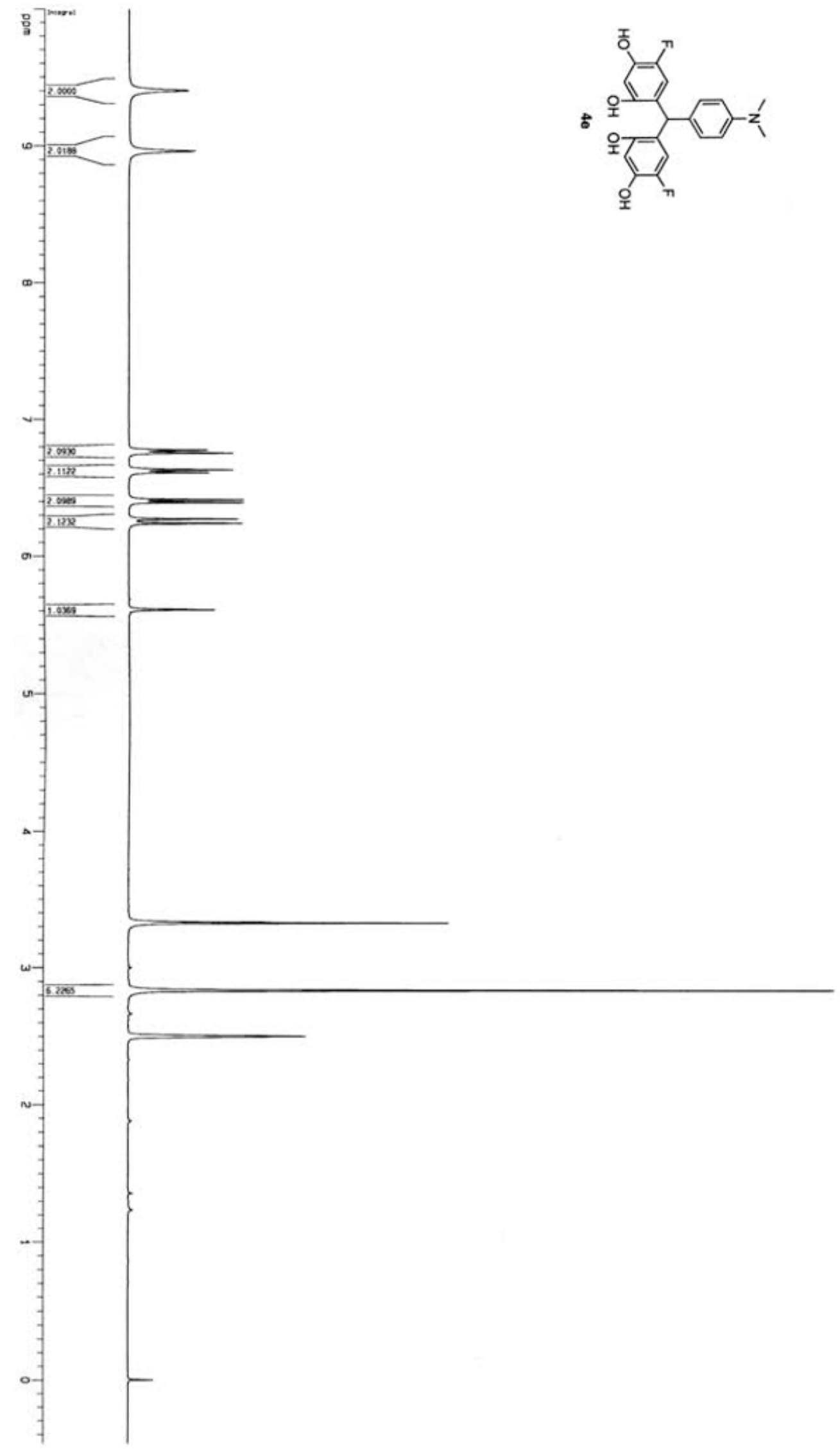




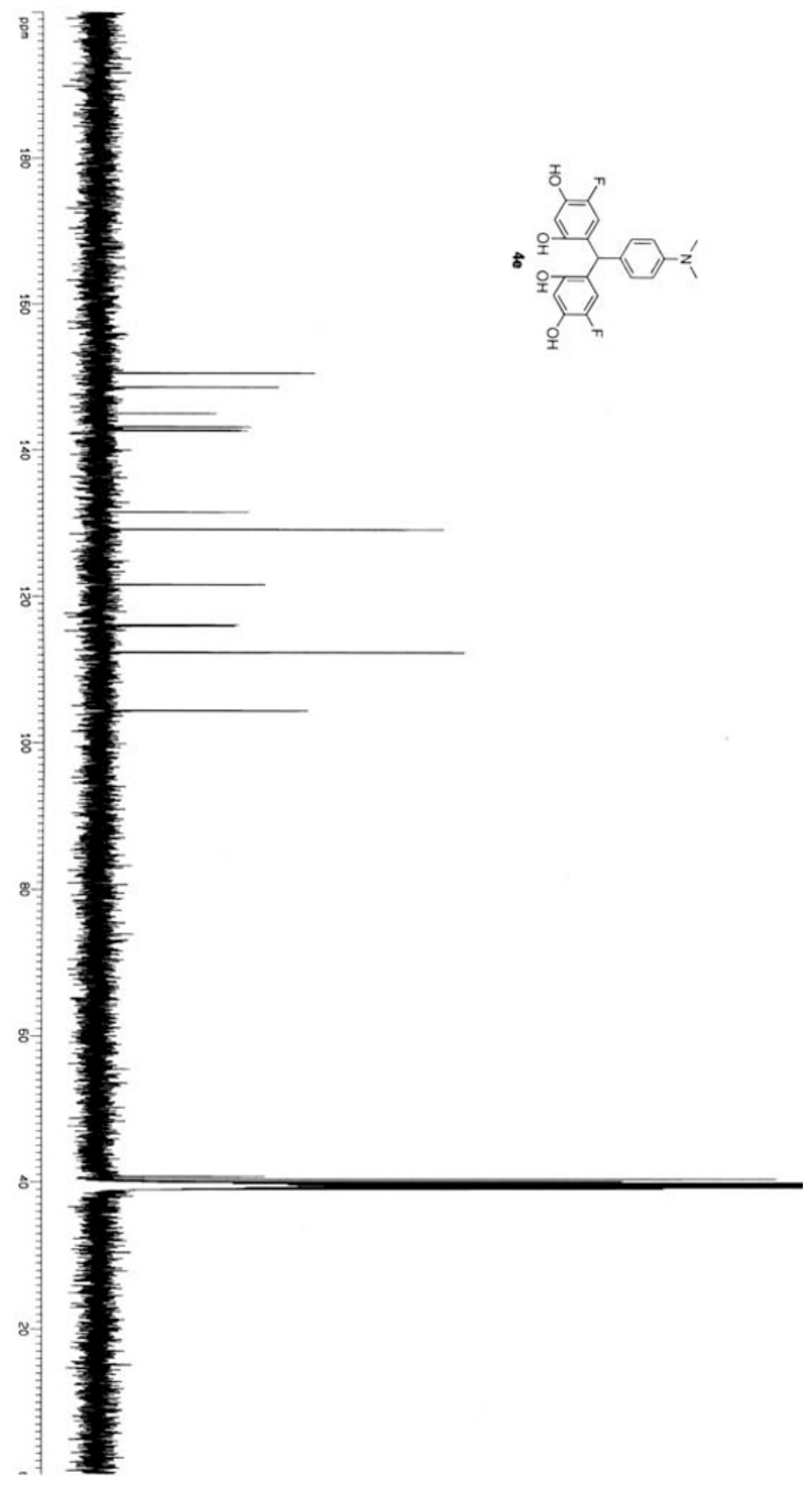




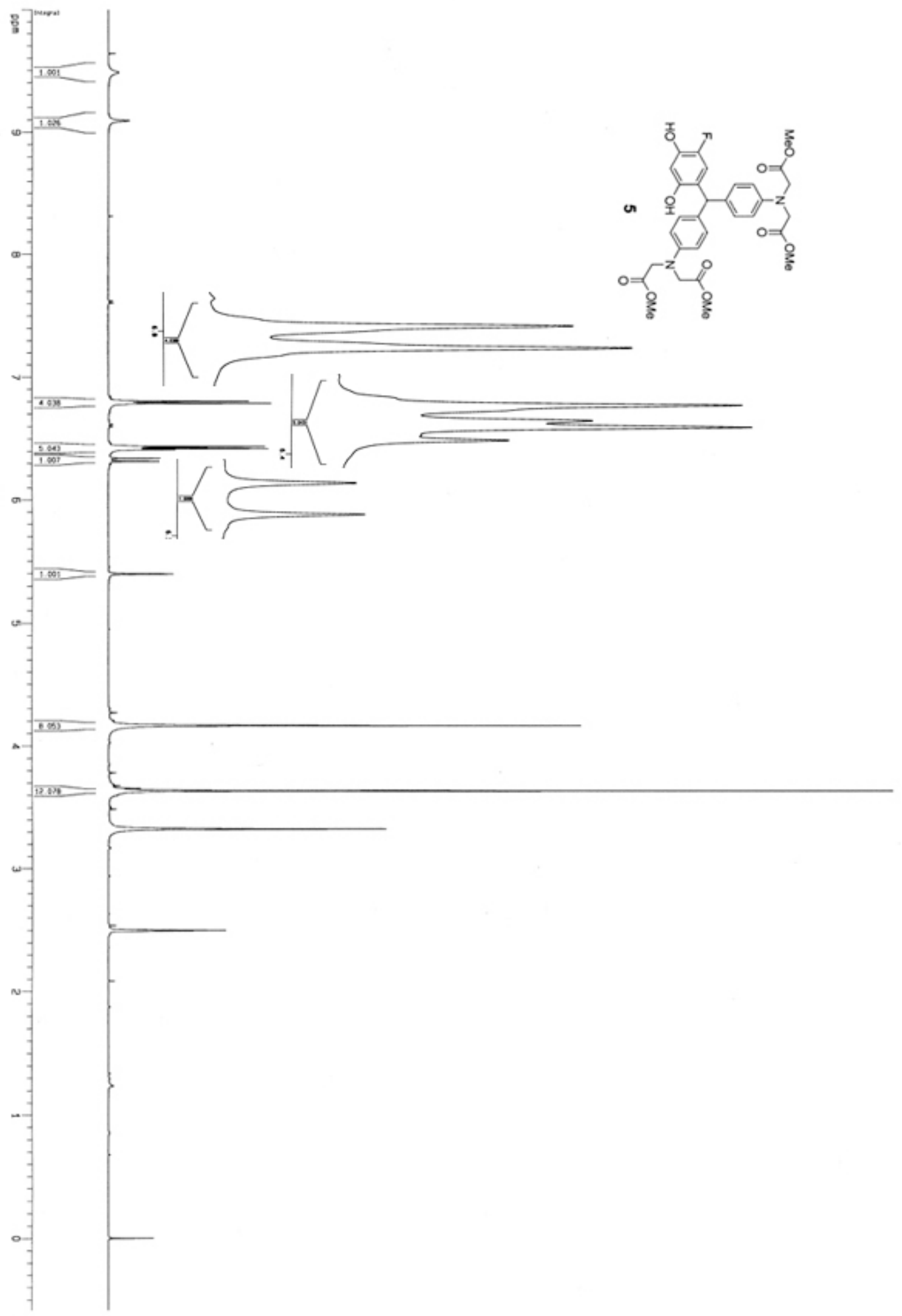




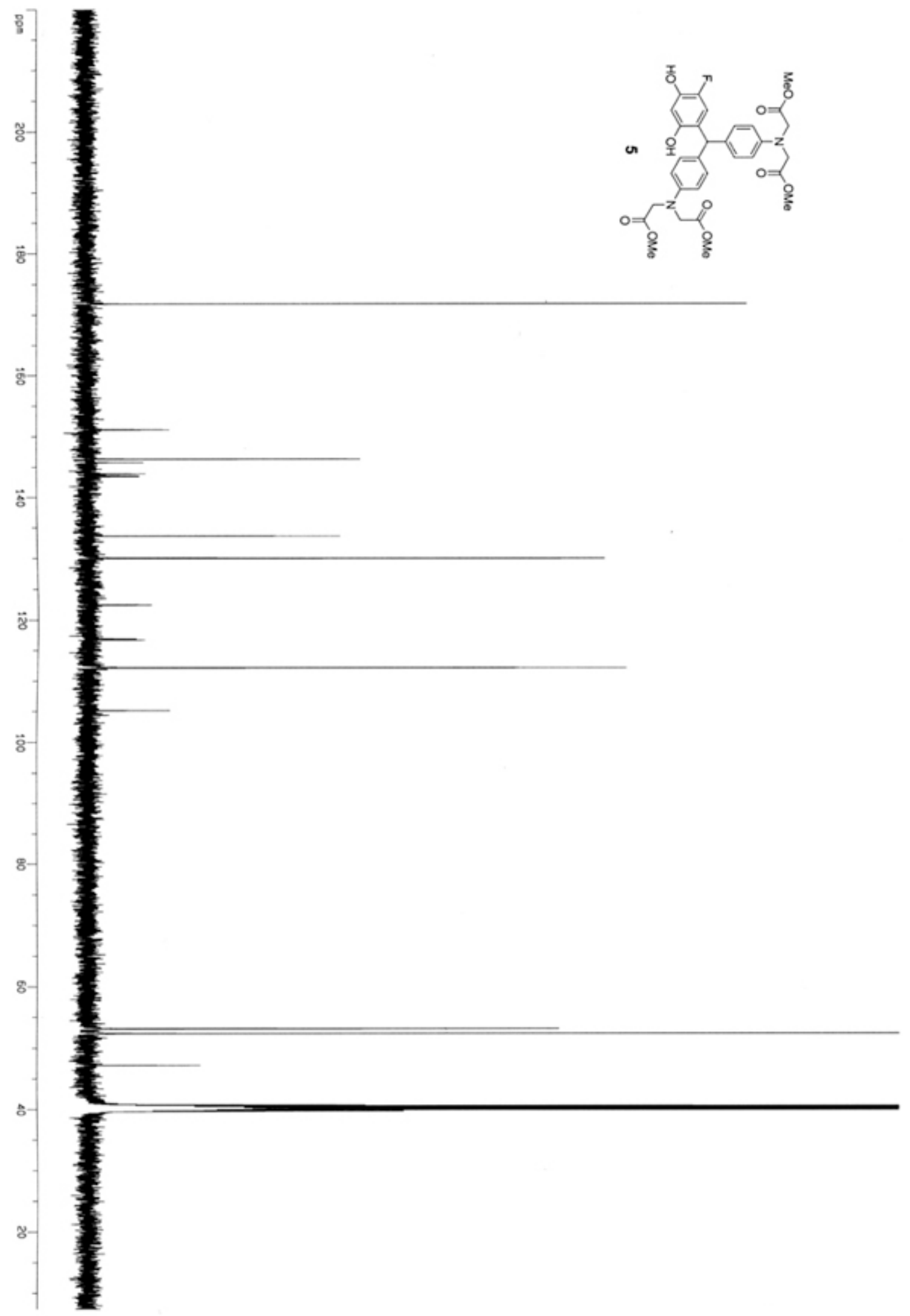




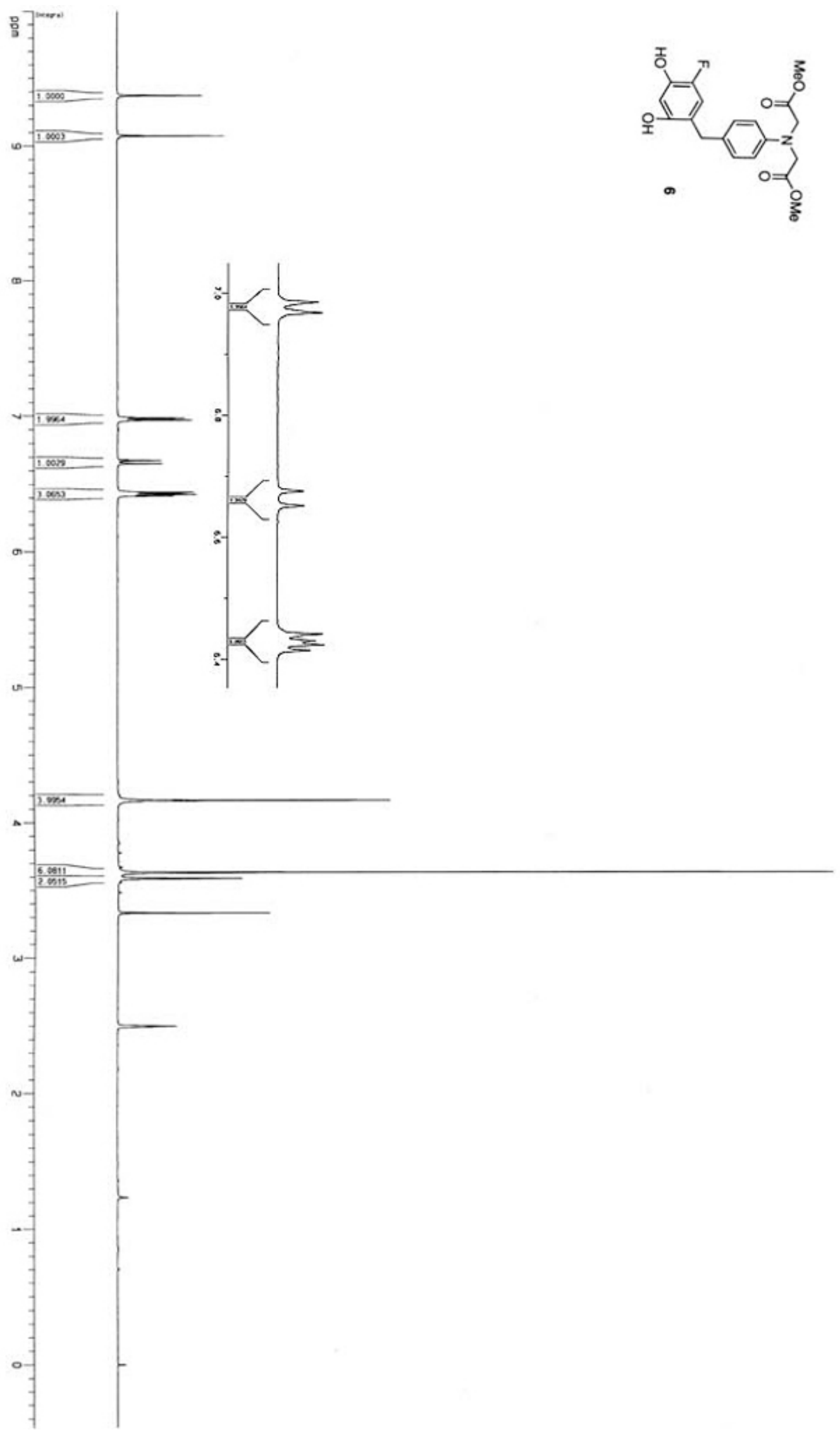




$$
\mid
$$




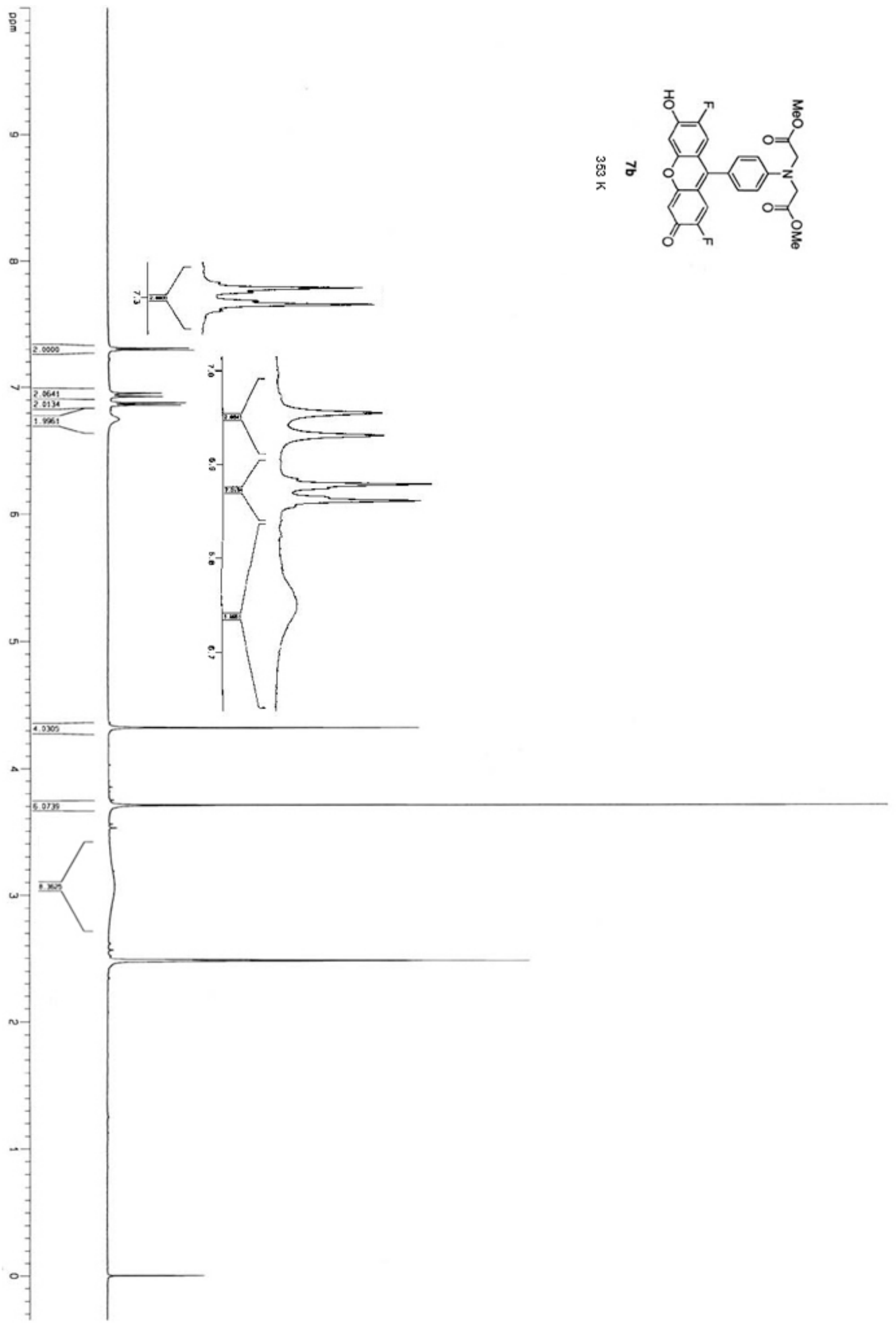




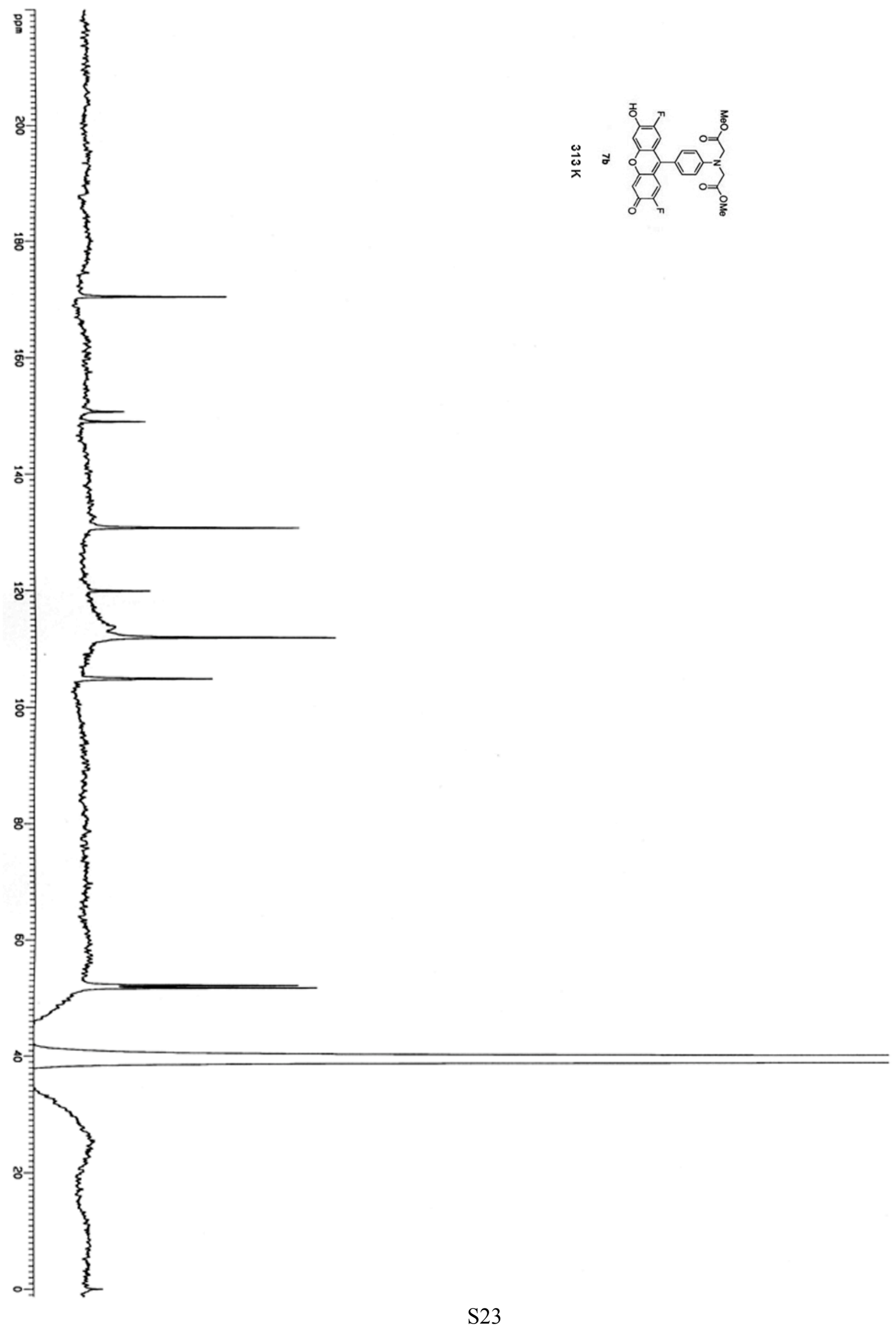




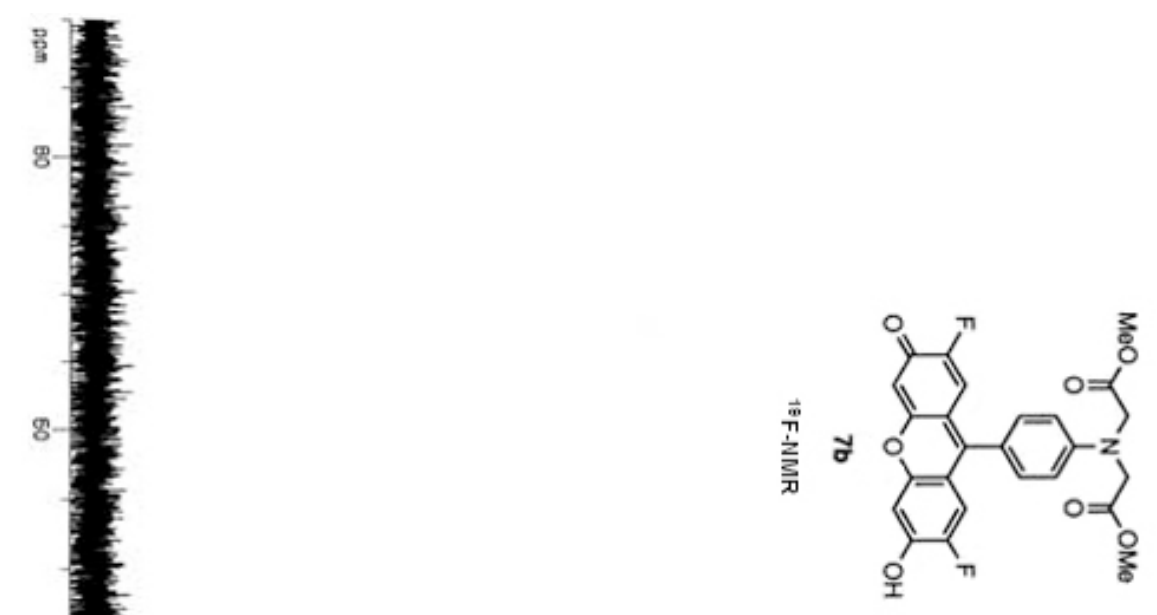




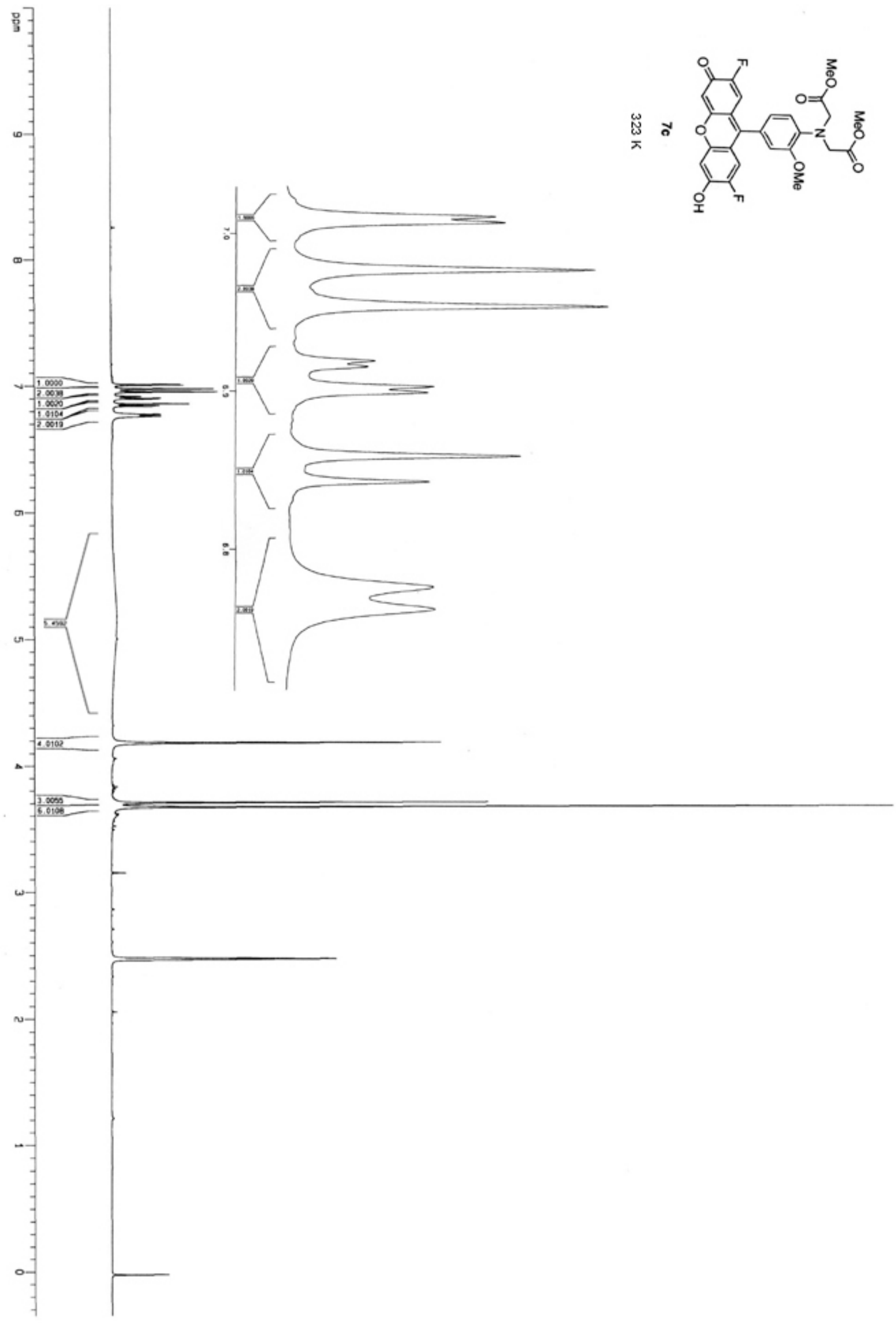




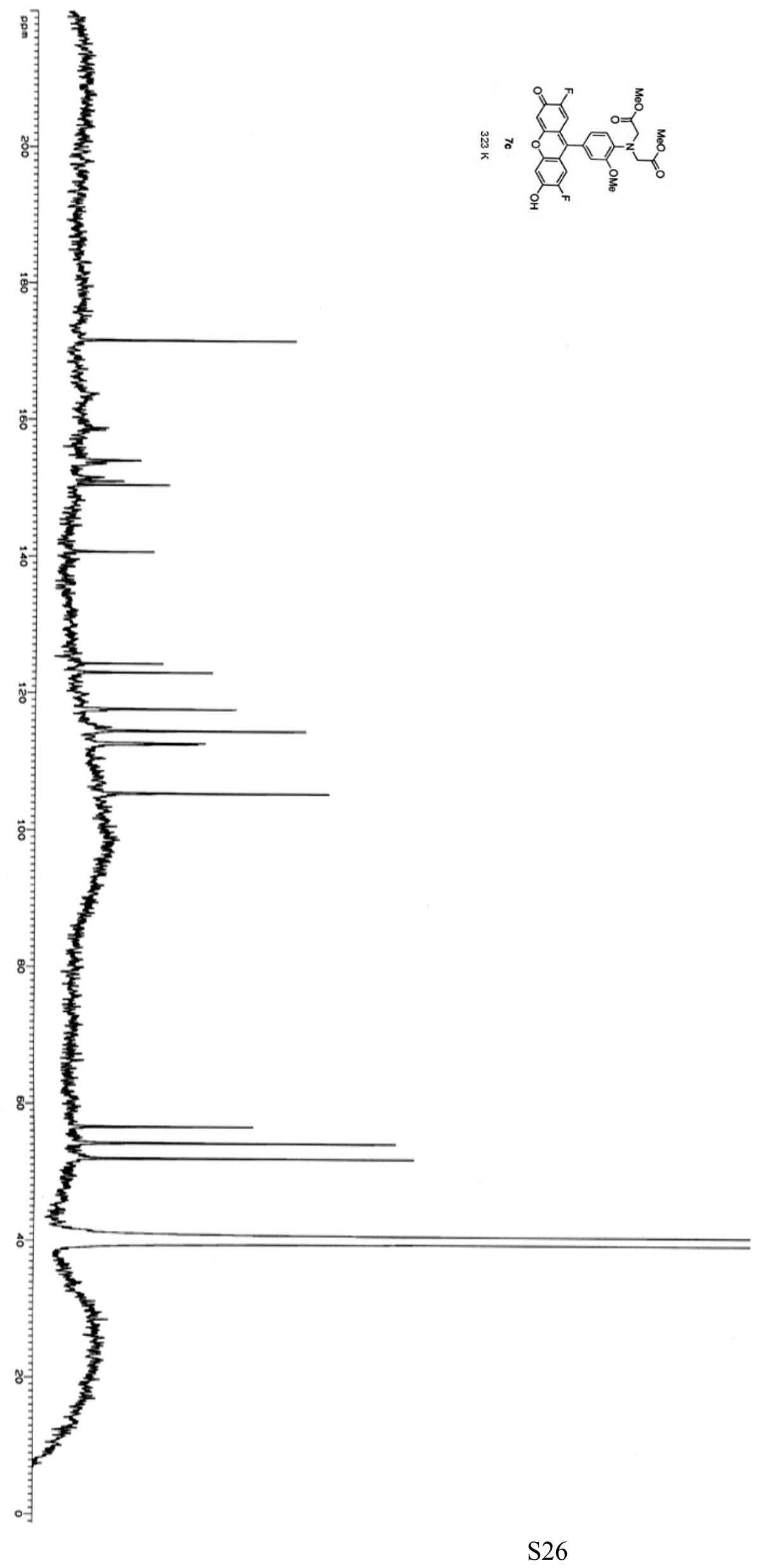




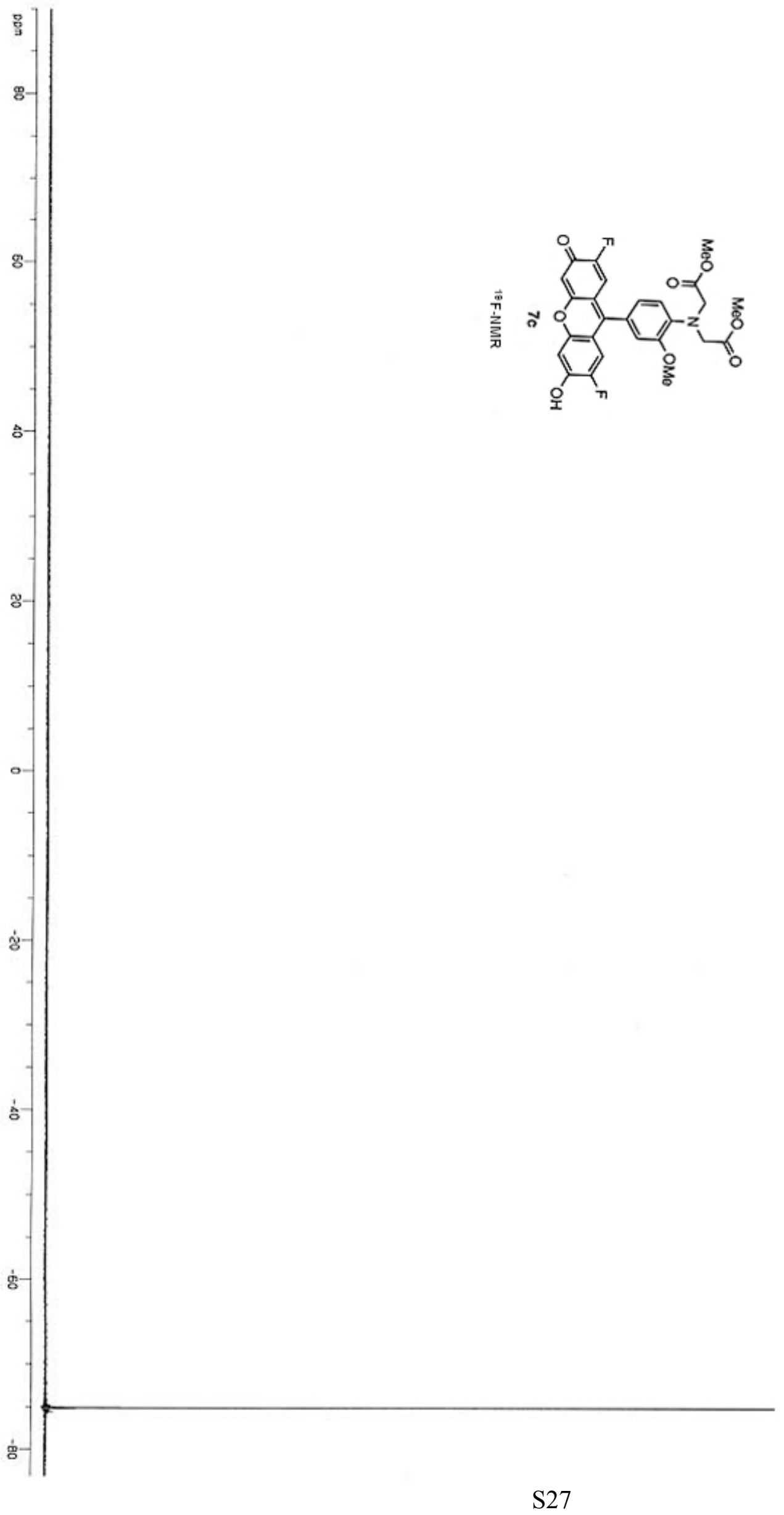




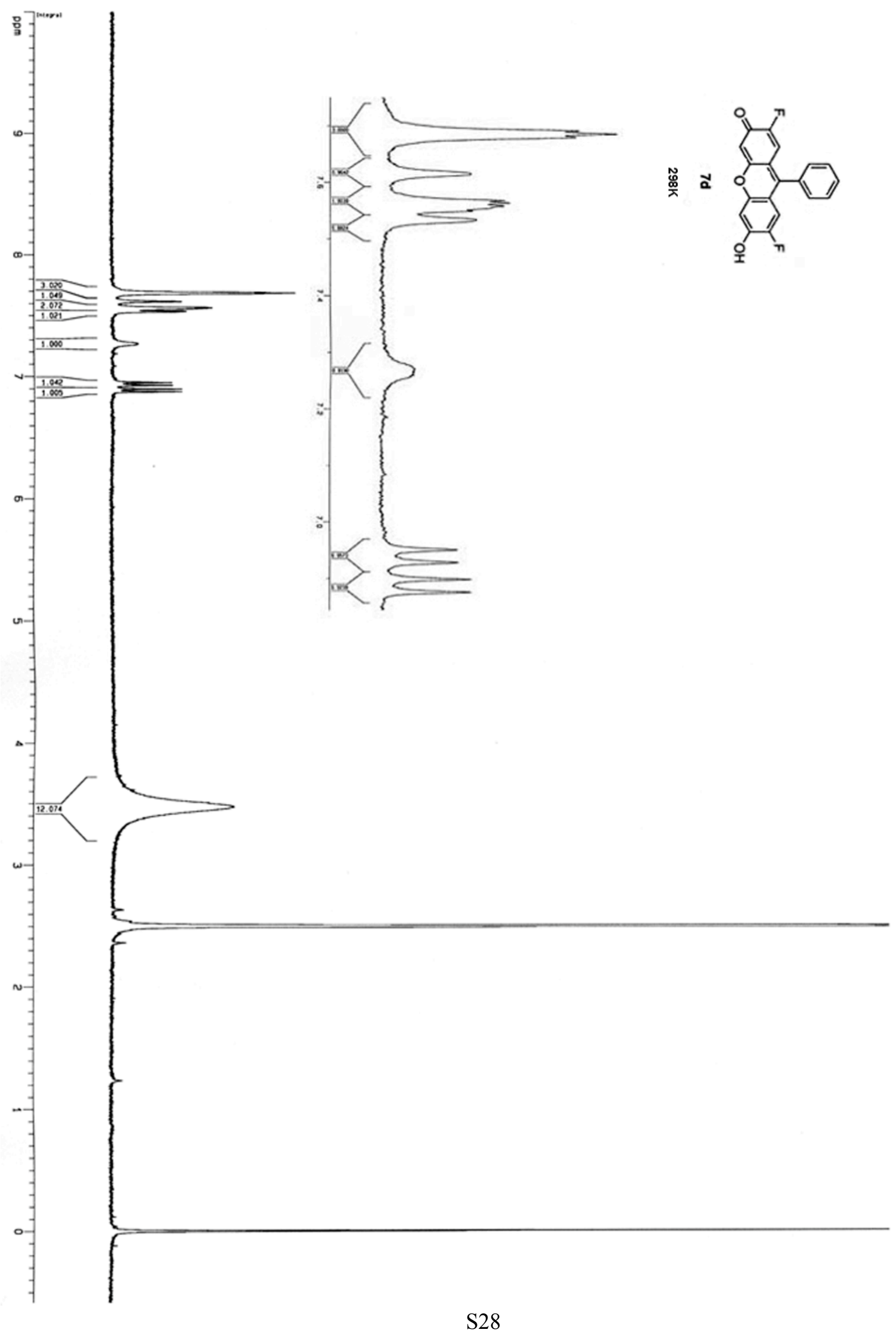




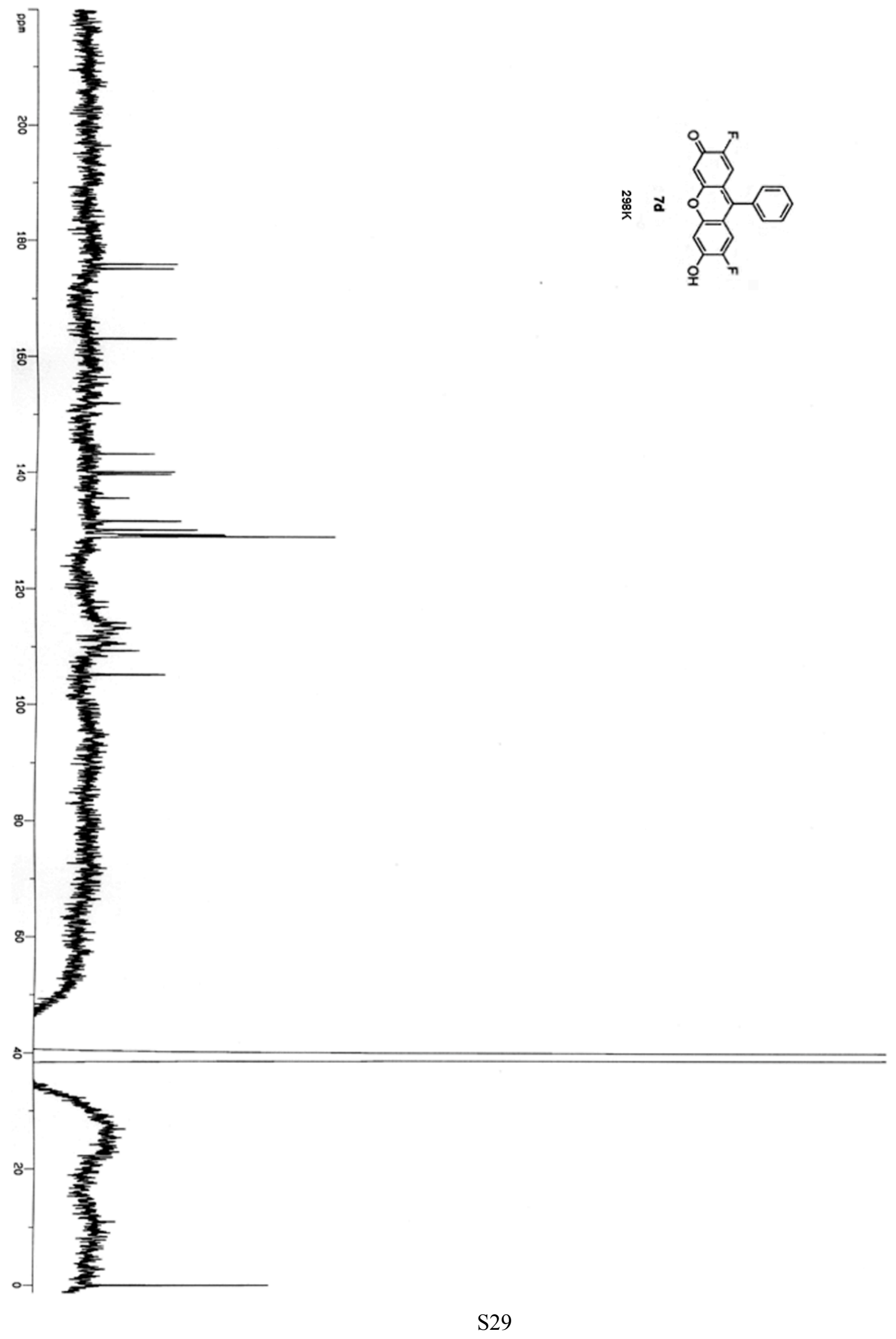




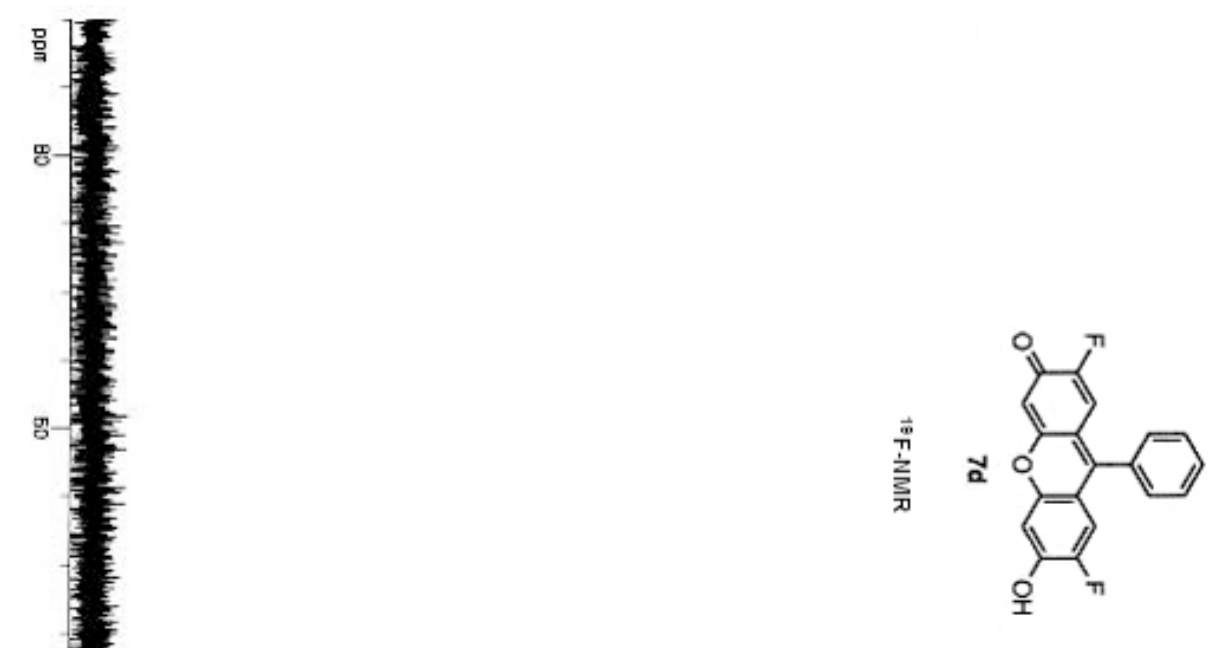

